RESEARCH ETHICS

\title{
Informed consent and participant perceptions of influenza vaccine trials in South Africa
}

\author{
K Moodley, M Pather, L Myer
}

J Med Ethics 2005;31:727-732. doi: 10.1136/jme.2004.009910

See end of article for authors' affiliations

....................

Correspondence to: Prof K Moodley, Bioethics Unit-Tygerberg Division,

PO Box 19063, Tygerberg 7505, South Africa; km@ sun.ac.za

Received 2 July 2004 In revised form

29 March 2005

Accepted for publication 4 April 2005
Background and objectives: There are few insights from sub-Saharan Africa on research participants' experiences of the informed consent process, particularly in the context of randomised controlled trials, where issues of randomisation and the use of placebos may be confusing concepts for participants. This study investigated the knowledge and perceptions of the informed consent process among individuals participating in influenza vaccine trials in two disadvantaged communities in South Africa.

Method: Four to 12 months after completion of the trials, participants were contacted to return to participate in the informed consent study. The semistructured questionnaire administered to assess recall of trial procedures and the informed consent process covered key issues including: purpose of the study; awareness that the study was not part of routine treatment; voluntary nature of participation and freedom to withdraw; randomisation; placebos; and remuneration.

Results: A total of 334 participants $(93 \%$ of the original vaccine trial sample; mean age 68 years, median level of education grade $8,69 \%$ women) completed the questionnaire. Only $21 \%$ were able to recall that they were allocated randomly to the different treatment arms. Only 19\% of those involved in the placebo controlled study had interpreted the concept of placebo as an inactive medication.

Conclusion: Although a good general recall of trial concepts was demonstrated, only a small proportion of the participants correctly interpreted and recalled the concepts of randomisation and placebos. Informed consent in this and similarly disadvantaged communities may often be inadequate and new ways to improve understanding of the research process should be explored.
$\mathrm{T}$ he randomised controlled trial (RCT) is the most widely accepted source of evidence for the safety, tolerability, and effectiveness of medical interventions. The main reason for this is scientific: properly conducted trials produce valid data which advance knowledge of optimal medical care. However in the process, the interests of participants may be unavoidably subjugated for the common good. ${ }^{12}$

\section{INFORMED CONSENT IN RANDOMISED CONTROLLED TRIALS}

Informed consent is fundamental to the ethical conduct of randomised controlled trials and is a critical component of the research process. Defined as "an autonomous authorization by individuals of a medical intervention or of involvement in research", ${ }^{3}$ the principle of informed consent is enshrined in all major guidelines for the ethical conduct of biomedical research. ${ }^{4-6}$ Informed consent is a process, based on verbal and written communication between participants and trial staff (or other individuals recruiting participants). The main pragmatic worry about informed consent is the different ways in which the process can fail-for example, because consent is not sought or because participants may not adequately understand the issues involved.

Written trial materials are a central component of the informed consent process that is required by most major ethical guidelines. ${ }^{4-6}$ To enhance understanding of informed consent forms and related patient information materials, it is essential that these documents are highly readable. Research in South Africa and other developing countries often uses relatively complex and detailed patient information leaflets written in academic language, sometimes imported from developed countries. This occurs despite concerns regarding the readability of informed consent documents in developed countries like the USA. ${ }^{7}$ In South Africa, there is the additional challenge of enrolling patients who are often educationally disadvantaged, as most biomedical research involves previously disadvantaged non-white population groups. In this research setting, one or more of the 11 official languages may be spoken, adding to the complexity of translation of consent documents to the consent process. Finding the most accurate words to explain research related concepts, such as placebo and randomisation, in each language is often challenging.

Conducting empirical research on the understanding of the informed consent process can be complex as a result of the confounding effect of recall in conducting a test of understanding. Differentiating between recall and understanding is a significant problem in informed consent research. ${ }^{8}$ This problem may be compounded by the timing of the test of understanding. Furthermore, there is a relation between severity of disease and retention of trial related information. ${ }^{9}$

Although the factors that may affect participants' understanding and recall of informed consent are likely to vary across research settings, there has been surprisingly little research investigating the comprehension and/or recall of informed consent in developing countries. Two South African studies have been published to date. The first study concluded that consent was informed but not always voluntary. ${ }^{10}$ The second study found that despite the participants having eight years of schooling or more, they had "poor knowledge about the most basic details of the trial". ${ }^{11}$ The authors concluded that subjects' participation could not be seen as informed. Regarding the voluntary nature of the consent, the same study found that although the respondents believed that their participation was voluntary they were clearly aware of the lack of alternative sources of care. ${ }^{11}$ Data on informed consent in other developing countries have focused mainly on willingness to participate in human immunodeficiency virus (HIV) vaccine trials. A hypothetical informed consent study conducted in 
Uganda found that trial related procedures like randomisation, placebos, and blinding were unfamiliar. ${ }^{12}$ An informed consent study conducted in Brazil, also on HIV vaccine trials, found that only $30 \%$ of the sample understood the concept of randomisation. ${ }^{13}$

To address the lack of empirical evidence regarding the assessment of informed consent in South Africa, we investigated the recall of informed consent procedures among participants in two industry sponsored influenza vaccine trials conducted in Cape Town, South Africa. We focused, in particular, on participants' recall of key aspects of trial design that were a focus of the informed consent process, as well as the determinants of participants' overall perceptions.

\section{METHODS \\ Study setting}

This study is based on two trials of an experimental intranasal influenza vaccine, sponsored by a major pharmaceutical firm, each conducted over a 12 month period during 2001 and 2002. The 2001 trial used an intranasal placebo as its control arm, while the 2002 trial was unblinded and based on comparison with an existing intramuscular (IM) vaccine. Participants were drawn from two impoverished non-white communities outside Cape Town, and were recruited through community meetings and word of mouth. All trial related contacts took place at the provincial day hospitals located in the communities. Interested individuals were eligible for the trials if they were over 60 years of age, agreed to the study procedures, and were gauged mentally competent by a score of 21 or higher on a standard Mini-Mental State Examination (MMSE). This is a short (eight item) quantitative examination that is commonly used to assess cognitive impairment and is widely used both internationally and in South Africa, particularly in geriatric populations. ${ }^{14}{ }^{15}$ In the USA, the median MMSE score for normal adults ranges from 29 for individuals with nine or more years of education to 22 for those with four or fewer years of education. ${ }^{16}$ As compensation for their involvement, R50.00 (approximately US\$8.30 or $€ 6.7$ ) was given to trial participants at each of three regularly scheduled study visits, and a further R20.00 (approximately US\$3.30 or €2.7) was provided to meet transport costs for unscheduled visits. These amounts are not considered a significant income supplement in this setting. ${ }^{17}$

\section{Informed consent procedure}

All participants received three separate explanations of the trial procedures prior to agreeing to participate. Community meetings during the recruitment phase included a discussion of the risks and benefits associated with the trial. Several weeks later, groups of interested individuals then discussed their participation with study nurses, which included a step by step verbal explanation of a patient information leaflet. The design of this leaflet was based on the International Conference on Harmonization guidelines for Good Clinical Practice. Participants were given the leaflet in their home language (Afrikaans or English) to take home, to re-read, and to discuss their participation with family members. Finally, on the day of enrolment into the trial, study doctors reviewed the leaflet again with the participants before written informed consent was provided. After this, participants were randomised during their enrolment visit using a scratch card system that allowed the trial assignment to take place in front of participants.

At the time of the study the authors (who were investigators on the influenza vaccine trials) were unaware of the application of readability scores to informed consent documents. We did, however, feel that the forms were written in academic language and that the trial information would be difficult for participants to understand. Hence, the detailed informed consent process outlined above in which enhanced verbal explanations were provided at community, group, and individual levels. Despite this, study personnel felt that comprehension may have been poor after enrolment so the information materials provided to trial participants were subsequently scored for readability using the Flesch readability formula and the Fry readability scale. Analysis of the patient information leaflet revealed a Flesch score of 46, which would fall into the "difficult" category, and this corresponded to a Fry score indicating a grade 12 reading level. ${ }^{18-20}$ After this assessment, the informed consent study described here was initiated by the clinical investigators at the two vaccine trial sites.

\section{Assessment of participants' perceptions of the informed consent process}

Four to 12 months after completion of the trial, participants were contacted to return to participate in the informed consent study. A semistructured questionnaire was designed to assess knowledge and perceptions of trial procedures and the informed consent process. The questionnaires covered awareness that the study constituted research and not routine treatment, an understanding of the purpose of the study, voluntary nature of participation and freedom to withdraw, interpretation of randomisation, use of placebos, and participant remuneration. Questions regarding the use of placebos were only administered to participants in the 2001 placebo controlled vaccine trial. Interviews lasted approximately 20 minutes and were conducted in participants' home language by a study nurse who was not affiliated with the trial site. Participants were assured by study staff that their involvement and responses would not affect their health care in any way.

\section{Data analysis}

We analysed the quantitative data using SAS (version 8.02, Cary, USA). Two simple scoring systems were developed a priori to gauge participants' overall understanding of the trial from the informed consent procedure. Firstly, a six item informed consent understanding score was constructed for all participants (from both the placebo and IM vaccine trials) using questions regarding informed consent: whether participation was part of standard medical care or a research project; what vaccine was being tested; whether participation was voluntary; whether the decision to participate would affect standard medical care; whether the participant could withdraw voluntarily from the study; and whether an effective 'flu vaccine was available to all participants as part of routine medical care. With this system participants could receive a maximum score of six if they correctly responded to each question, and a minimum score of zero.

Secondly, a separate placebo understanding score, based on four items, was administered to only participants in the 2001 placebo controlled trial. The items included: whether the participant understood the concept of randomisation by chance; whether the participant understood the concept of "inactive medicine"; whether they correctly stated that "inactive medicine" meant no effective treatment; and whether they understood that their trial assignment was determined by chance (rather than by a study doctor, nurse, or the participant's own decision). The range of possible scores was from four to zero.

Data were summarised using means, medians, and proportions. Throughout the analysis, the correlation coefficients $(r)$ and Student's $t$ tests were used to examine the relation between understanding scores and participant characteristics, and $\chi^{2}$ tests were used to compare the proportions of subjects answering each question correctly. Multiple linear regression was used to estimate the effect of 
Table 1 Participant responses to informed consent related questions $(n=334)$

\begin{tabular}{|c|c|c|}
\hline \multirow{2}{*}{$\begin{array}{l}\text { Question } \\
\text { Was your participation in the trial part of usual treatment, or a } \\
\text { research project?* }\end{array}$} & \multicolumn{2}{|l|}{ Responses $(\%) \dagger$} \\
\hline & Usual treatment & $15(5)$ \\
\hline \multirow{3}{*}{ What experimental vaccine was being tested?* } & Research project & $318(95)$ \\
\hline & $\begin{array}{l}\text { Injectable/intramuscular } \\
\text { vaccine }\end{array}$ & $36(11)$ \\
\hline & Nasal spray vaccine & $297(89)$ \\
\hline \multirow{4}{*}{$\begin{array}{l}\text { Who or what determined what vaccine you would receive as part } \\
\text { of the study?* }\end{array}$} & Doctor & $121(37)$ \\
\hline & Study nurse & $75(23)$ \\
\hline & Participant chose for self & 60 (19) \\
\hline & Chance/randomisation & $67(21)$ \\
\hline \multirow{2}{*}{$\begin{array}{l}\text { Did you feel that you received a clear explanation of the trial } \\
\text { procedures? }\end{array}$} & Yes & $327(98)$ \\
\hline & No/unsure & $7(2)$ \\
\hline \multirow{2}{*}{ Did you read the information leaflet that was given to you? } & Yes & $322(96)$ \\
\hline & No/unsure & $12(4)$ \\
\hline \multirow[t]{2}{*}{ Was the information leaflet easy to understand? } & Yes & $310(93)$ \\
\hline & No/unsure & $22(7)$ \\
\hline \multirow[t]{2}{*}{ Was your participation in the trial compulsory?* } & Yes & $1(<1)$ \\
\hline & No & $331(>99)$ \\
\hline \multirow{2}{*}{$\begin{array}{l}\text { If you chose not to participate in the trial, would your usual health } \\
\text { care have been affected?* }\end{array}$} & Yes/unsure & $41(12)$ \\
\hline & No & $293(88)$ \\
\hline \multirow[t]{2}{*}{$\begin{array}{l}\text { If you chose not to participate in the trial, what alternative } \\
\text { treatment could you have received? }\end{array}$} & $\begin{array}{l}\text { No alternative treatment } \\
\text { available }\end{array}$ & $141(42)$ \\
\hline & $\begin{array}{l}\text { 'Flu injection at a general } \\
\text { practice or hospital }\end{array}$ & $191(58)$ \\
\hline \multirow{2}{*}{$\begin{array}{l}\text { Were you aware that you could withdraw from the trial at any } \\
\text { stage if you wanted to?* }\end{array}$} & Yes & $289(87)$ \\
\hline & No/unsure & $44(11)$ \\
\hline \multirow[t]{2}{*}{ Did you wish to withdraw from the trial at any stage? } & Yes & $4(1)$ \\
\hline & No/unsure & $328(99)$ \\
\hline \multirow[t]{2}{*}{ Would you participate in a research study of this type again? } & Yes & $322(96)$ \\
\hline & No/unsure & $12(4)$ \\
\hline \multicolumn{3}{|c|}{$\begin{array}{l}\text { Questions that were included in the informed consent comprehension score are marked with an asterix (*), and th } \\
\text { responses that were considered correct for the purposes of the informed consent comprehension scale are } \\
\text { highlighted in bold. } \\
\text { †For some items the number of responses sums to slightly less than the total number of participants as not all } \\
\text { participants completed all items. }\end{array}$} \\
\hline
\end{tabular}

participant characteristics on the scores achieved for each scale. All models reported here were adjusted for age, level of education, and MMSE score, each modelled as a continuous variable. The coefficients $(\beta)$ from these models can be interpreted as adjusted differences in mean scores, with a 1 unit change in the independent variable (that is, age, education, MMSE) corresponding to a $\beta$ unit change in the mean score on the scale in question. Model fit was assessed using standard diagnostic procedures, ${ }^{21}$ and all statistical tests were two sided at $\alpha=0.05$.

\section{Ethical approval}

Each participant provided written consent, separate from the consent for participation in the vaccine trial, before being interviewed for this study. The University of Stellenbosch Pharmaceutical Trials Ethics Committee provided ethical approval for this study, as well as the two influenza vaccine trials on which this research was based.

\section{RESULTS}

A total of 334 trial participants completed the informed consent questionnaire, 131 from the trial of nasal spray versus placebo and 203 from the trial of nasal spray versus IM vaccine. This sample represented $93 \%$ of subjects from the vaccine trials returning for the informed consent study. The mean age of participants was 68 years (range: 60-80) and $69 \%$ of the sample were women. The median level of education was grade 8 (range: none to grade 12 ) and the mean MMSE score was 26.5 (range: 21-30).

\section{Participants' perceptions of informed consent}

Table 1 shows the participant responses to each of the informed consent questions. A high proportion of participants knew that the trial was not part of their usual treatment, that the vaccine being tested was experimental, and that they could withdraw from the trial at any time. However, most subjects thought that their trial assignment was chosen by a study doctor or nurse, while only a fifth of subjects thought that their assignment was determined by chance only.

The mean (SD) score on the six items which comprised the informed consent scale was 5.1 (0.8). No participant answered every question incorrectly, and a third of the participants $(n=109)$ responded correctly to each question. In bivariate analysis, participants' scores on this scale were positively correlated with increasing level of education $(r=0.15 ; \mathrm{p}<0.01)$ and MMSE score $(r=0.18 ; \mathrm{p}<0.01)$, as well as with decreasing age $(r=-0.12 ; \mathrm{p}=0.03)$. When entered into a multiple linear regression model, MMSE score $(\beta=0.05 ; p=0.05)$ and age retained slight associations with comprehension of the informed consent process, although the association with educational level did not persist.

\section{Participants' perceptions of placebo}

The responses of the 131 participants in the placebo controlled trial to the placebo related items are presented in table 2. Less than half of those interviewed could recall the concept of an inactive placebo, and that their trial assignment was determined by chance. Only $19 \%$ indicated that a placebo involved receiving no active medicine, and more than a third 
Table 2 Participant responses to placebo trial related questions $(n=131)$

\begin{tabular}{|c|c|c|}
\hline \multirow{2}{*}{$\begin{array}{l}\text { Question } \\
\text { Did you understand that you had the same } 50 \% \text { chance of } \\
\text { receiving the inactive medicine (placebo) or the active medicine } \\
\text { (experimental vaccine)?* }\end{array}$} & \multicolumn{2}{|l|}{ Responses $(\%) \dagger$} \\
\hline & Yes & $64(49)$ \\
\hline & No/not sure & $67(51)$ \\
\hline \multirow[t]{2}{*}{$\begin{array}{l}\text { Do you think you understand the meaning of "inactive medicine" } \\
\text { (placebo)?* }\end{array}$} & Yes & $46(37)$ \\
\hline & No/not sure & 77 (63) \\
\hline \multirow[t]{5}{*}{ What does "inactive medicine" (placebo) mean?" } & Nasal spray & $50(38)$ \\
\hline & No vaccine & 25 (19) \\
\hline & Weaker vaccine & $12(9)$ \\
\hline & Different type of vaccine & $2(2)$ \\
\hline & Not sure/other & $42(32)$ \\
\hline \multirow{2}{*}{$\begin{array}{l}\text { Would you continue in the study if you knew you may not } \\
\text { receive the vaccine? }\end{array}$} & Yes & $80(62)$ \\
\hline & No/not sure & 49 (38) \\
\hline \multirow{4}{*}{$\begin{array}{l}\text { Who decided whether you would receive active medicine } \\
\text { (vaccine) or inactive medicine (placebo)?* }\end{array}$} & Doctor & $52(40)$ \\
\hline & Nurse & $23(18)$ \\
\hline & Participant chose for self & $39(30)$ \\
\hline & Chance/randomisation & 15 (12) \\
\hline \multirow{3}{*}{ Which treatment do you think you received? } & Vaccine & $83(64)$ \\
\hline & Placebo & $4(3)$ \\
\hline & Not sure & $43(33)$ \\
\hline \multicolumn{3}{|c|}{$\begin{array}{l}\text { Questions that were included in the placebo comprehension score are marked with an asterix }\left({ }^{*}\right) \text {, and the } \\
\text { responses that were considered correct for the purposes of the placebo comprehension scale are highlighted in } \\
\text { bold. } \\
\text { tFor some items the number of responses sums to slightly less than the total number of participants as not all } \\
\text { participants completed all items. }\end{array}$} \\
\hline
\end{tabular}

of the participants $(38 \%)$ stated that they would not have continued to participate in the trial if they had known that they had received an inactive placebo.

The mean score on the four item placebo comprehension scale was 1.2 (1.1). One participant answered all four items correctly, whereas 45 participants (34\%) did not answer any of the items correctly. This score did not appear to be associated with participant age, but was strongly correlated with increasing level of education $(r=0.24 ; \mathrm{p}<0.01)$ and higher MMSE scores $(r=0.28 ; \mathrm{p}<0.01)$. In multivariate analysis adjusted for educational level and participant age, MMSE score remained correlated with placebo comprehension $(\beta=0.12 ; p=0.03)$.

\section{Participant remuneration}

Most participants received a total of R150.00 (approximately US $\$ 25$ or $€ 20$ ) in remuneration over the course of the trial. When asked whether the remuneration they received was adequate, 281 subjects $(84 \%)$ were satisfied with R50.00 (approximately US\$8.30 or €6.7) per visit ${ }^{17}$ whereas 49 subjects $(16 \%)$ either stated that the amount was too little or were unsure; no participant stated that the amount was too much. Participants who felt that the amount they received was too little, or were unsure of the amount, had higher recall scores on both the informed consent and placebo scales compared with the participants who felt that the amount they received was enough (mean informed consent recall score $5.4 \vee 5.0$, respectively, $\mathrm{p}=0.01$; mean placebo recall score of $1.5 \vee 1.1$, respectively, $\mathrm{p}=0.14)$. The association between perceptions of the amount of remuneration and informed consent recall persisted after multivariate adjustment for participant age, education, and MMSE score (mean difference in informed consent score, those who felt the amount received was inadequate or unsure $v$ those who felt the amount was enough, $0.30, \mathrm{p}=0.02$ ).

\section{DISCUSSION}

An assessment of the informed consent process requires the conduct of a test of understanding of the basic elements of consent $^{22}$ as well as trial related concepts and procedures.
However, it is often very difficult to draw a distinction between understanding and recall of trial information. ${ }^{23}$ An attempt has been made to distinguish a test of recall from a test of understanding in the form of the Deaconess Informed Consent Comprehension Test, but this method remains insufficiently tested to draw conclusions regarding reliability and validity.$^{24}$ In the absence of a precise method to measure understanding, and given the fact that understanding and recall are interrelated in the cognitive processing of trial related information especially if the assessment is conducted months after the enrolment of study participants, we have focused on recall of trial related information in this study. ${ }^{25}$

Participants in these influenza vaccine trials demonstrated a good general recall of the informed consent process. However, the present results show low levels of recall surrounding two of the most complex-and arguably the most important-aspects of randomised controlled trial participation: randomisation and the concept of placebo.

\section{Trial participants' understanding of randomisation and placebos}

Only a small fraction of trial participants recognised that their treatment allocation was determined by chance alone, rather than by study personnel. This is consistent with the findings of Featherstone and Donovan, who reported that most patients interviewed found the concept of randomisation difficult to accept, suggesting that the term "random" has different meanings to lay and professional audiences. ${ }^{26}$ Similarly, Snowdon et al indicated how difficult it can be for patients to make sense of a treatment decision that is based on chance. ${ }^{27}$ This is so because patients are traditionally accustomed to treatment decisions being taken in their best interests. The scientific method that incorporates concepts like randomisation and blinding is often incompatible with "personal care", a concept that requires that the doctor's first obligation is solely the wellbeing of the patient. ${ }^{28}$ In fact, one of the consequences of a misunderstanding of the concept of randomisation is a failure to appreciate the distinction between the goals of clinical research and ordinary treatment-the therapeutic misconception. ${ }^{29}{ }^{30}$ Under the 
therapeutic misconception, participants fail to appreciate their chances of not receiving any treatment at all if randomised to a placebo group or of merely receiving experimental, unproved treatment if allocated to the study arm of the trial.

Most participants in the placebo controlled trial did not recall that assignment to the placebo arm would involve receiving no active treatment. Several factors require consideration when interpreting these results. Firstly, with a mean age of 68 years, our study population was substantially older than research subjects in many studies. However, research focusing on geriatric populations is becoming increasingly common, and it is important to understand the perceptions of informed consent in this group. ${ }^{31-33}$ Secondly, our results are based on a follow up interview that took place up to 12 months after the influenza vaccine trials were completed. This relatively long delay between trial participation and informed consent assessment is a limitation of the study. However, it may mean that levels of comprehension were higher during the trial, although we found no differences in participants' recall according to the time elapsed between trial participation and undertaking this study. A previous study on informed consent involving research participants 60 years and older had found similar results using a test of comprehension administered soon after the informed consent process. ${ }^{34}$ Schaeffer et al assessed the impact of disease severity on retention of trial related information. The most relevant finding of their study was that changes in recall and understanding do not always decrease over time. ${ }^{9}$ Finally, while the recall of informed consent was based on factual knowledge, several of the questions we used in this study involved participants' subjective judgements regarding trial participation-in turn introducing the possibility of social desirability biases from participants who wish to please trial personnel. We made every effort to minimise this possibility by assuring participants that their responses would not affect their health care in any manner and by using interviewers who were not affiliated with the trial.

It is important to note that these results were observed despite an informed consent process that was relatively intensive for research studies conducted in developing countries. Prior to enrolment into the trial, participants had three separate contacts with study personnel, including two separate verbal explanations of the patient information leaflet. Study site coordinators read through the patient information leaflet one paragraph at a time with groups of five participants each, taking questions and providing lay explanations for each paragraph of the nine page patient information leaflet. These explanations were conducted in the participants' home language. Investigators at all three sites also provided verbal explanations in layman's terms of study related procedures. These efforts to help ensure an adequate explanation of the trial for all participants were more intensive than the informed consent process involved in most clinical and epidemiological research in this context, where participants may receive only a single explanation of the research study before being asked to participate. In this light, it is possible that in most research settings in South Africa and similar settings, participants' comprehension of the informed consent process may be substantially lower than these results suggest. On the other hand, the difficult readability level of the patient information leaflet could have resulted in the lower scores on recall of trial related information and this could represent a limitation of this study.

\section{Future directions}

Given the lack of empirical research to date investigating informed consent comprehension, coupled with the diversity of research settings across the developing world, further research on this topic is clearly needed. Simple evaluations of informed consent comprehension can be critical in designing more effective participant education materials, particularly in instances where relatively complex concepts such as randomisation and placebos are involved. For example, Rikkert et al describe a process of "experienced consent" in a group of elderly research participants who were subjected to a mock trial before being asked to provide consent for participation in the actual trial. ${ }^{31}$ Fitzgerald et al in their study on comprehension of informed consent in Haiti advise that formal assessment of a research participant's comprehension of the consent form should be a routine step in the informed consent process in developing countries. ${ }^{35}$

\section{Summary}

This study suggests that participants' recall of informed consent in randomised controlled trials in South Africa and other developing countries may often be inadequate. Difficult and lengthy patient information leaflets impact negatively on understanding of trial related information. Hence, interventions to improve understanding of the research process, especially the concepts of randomisation and placebos, require greater attention. Given the limitations of this study in assessing recall rather than understanding, future research involving a test of understanding immediately after trial related information is presented to potential participants is indicated. Although this study is constrained by its ability to assess recall rather than understanding, it represents an important milestone in empirical research into the consent process in South Africa and has identified gaps in information retained by research participants.

\section{ACKNOWLEDGEMENTS}

We would like to acknowledge I Joseph and D Gallant who assisted in data collection at the sites. Professor PJT de Villiers, Principal Investigator of the influenza vaccine study and head of the Department of Family Medicine, University of Stellenbosch, provided valuable support during this study.

\section{Authors' affiliations}

K Moodley, Bioethics Unit, Centre for Applied Ethics and Department of Family Medicine and Primary Care, Faculty of Health Sciences,

University of Stellenbosch, South Africa

M Pather, Department of Family Medicine and Primary Care, University of Stellenbosch, South Africa

L Myer, School of Public Health and Family Medicine, University of Cape Town, South Africa

This study was funded by a research grant from the Department of Family Medicine and Primary Care, University of Stellenbosch.

\section{REFERENCES}

1 Lilford RJ, Jackson JC. Equipoise and the ethics of randomized controlled trials. J Roy Soc Med 1995;88:552-92.

2 Edwards SJL, Lilford RJ, Hewison J. The ethics of randomised controlled trials from the perspectives of patients, the public, and health care professionals. BMJ 1998;371:1209-12.

3 Beauchamp TL, Childress JF. Principles of Biomedical Ethics, 4th edn. New York: Oxford University Press, 1994.

4 The World Medical Association. Declaration of Helsinki October 2000. Available at www.wma.net/e/policy/b3.htm (accessed 1 October 2003).

5 International Conference on Harmonisation, Good Clinical Practice: Consolidated Guideline, May 1997. Available at www.fda.gov/cder/ guidance/iche6.htm (accessed 1 October 2003).

6 Guidelines for Good Clinical Practice in the conduct of Trials in Human Participants in South Africa. Pretoria: Department of Health, 1999. Available at: http://196.36.153.56/doh/docs/policy/trials/trials-full.html (accessed 1 October 2003)

7 Paashe-Orlow MK, Taylor HA, Brancati FL. Readability standards for informed consent forms as compared with actual readability. N Engl J Med 2003:348:721-6.

8 Rosenberg JD. Informed consent and sham surgery as a placebo in fetal cell transplant research for Parkinson's disease. Einstein Q J Biol Med 2003;20:14-18. 
9 Schaeffer MH, Krantz DS, Wichman A, et al. The impact of disease severity on the IC process in clinical research. Am J Med 1996;100:261-8.

10 Abdool Karim Q, Abdool Karim SS, Coovadia HM, et al. Informed consent for HIV testing in a South African hospital: is it truly informed and truly voluntary? Am J Public Health 1998;88:637-40.

11 Joubert G, Steinberg H, van der Ryst E, et al. Consent for participation in the Bloemfontein vitamin A trial: how informed and voluntary? Am J Public Health 2003:93:582-4.

12 McGrath JW, George K, Svilar G, et al. Knowledge about vaccine trials and willingness to participate in an HIV/AIDS vaccine study in the Ugandan military. J Acquir Immune Defic Syndr 2001;27:381-8.

13 Quieroz da Fonseca O, Lie RK. Informed consent to preventive AIDS vaccine trials in Brazil: A pilot study. AIDS Public Policy J 1995;10:22-6.

14 Tombaugh TN, Mclntyre NJ. The Mini-Mental State Examination: a comprehensive review. J Am Geriatr Soc 1992;40:922-35.

15 Lenger V, de Viliers C, Louw SJ. Informant questionnaires as screening measures to detect dementia. A pilot study in the South African context. S Afr Med J 1996;86:737-41

16 Crum RM, Anthony JC, Bassett SS, et al. Population-based norms for the MiniMental State Examination by age and educational level. JAMA 1993;269:2386-91.

17 Moodley K, Myer L. Participant remuneration for research-how much is enough? S Afr Med J 2003;93:677-8.

18 Fry E. A readability formula that saves time. J Reading 1968;11:513-16; 575-8.

19 Grundner TM. Two forms for determining the readability of subject consent forms. Am Psychol 1978;33:773-5.

20 Morrow GR. How readable are subject consent forms? JAMA 1980;244:56-8.

21 Cohen J, Cohen P, West SG, et al. Applied multiple regression/correlation analysis for the behavioral sciences. Mahwah, NJ: Lawrence Erlbaum Associates, 2003
22 Department of Health and Human Services. Federal Register 56 (18 June 1991); 45CFR 46.101-46.124.

23 Lindegger G, Richter LM. HIV vaccine trials: critical issues in informed consent. S Afr J Sci 2000:96:313-17.

24 Miller CK, O'Donnell DC, Searight HR, et al. The Deaconess informed consent comprehension test: an assessment tool for clinical research subjects. Pharmacotherapy 1996;16:872-8.

25 Fortney JA. Assessing recall and understanding of informed consent in a contraceptive clinical trial. Stud Fam Plann 1999;30:339-46.

26 Featherstone K, Donovan JL. Random allocation or allocation at random? Patients' perspectives of participation in a randomised controlled trial. BMJ 1998;317:1 177-80

27 Snowdon C, Garcia J, Elbourne D. Making sense of randomization; responses of parents of critically ill babies to random allocation of treatment in a clinical trial. Soc Sci Med 1997:45:1337-55.

28 Appelbaum PS, Roth LR, Lidz CW, et al. False hopes and best data: consent to research and the therapeutic misconception. Hastings Cent Rep 1987:20-4.

29 Lidz CW, Appelbaum PS. The therapeutic misconception: problems and solutions. Med Care 2002:40:55-63.

30 Molyneux CS, Peshu N, Marsh K. Understanding of informed consent in a low-income setting: three cases studies from the Kenyan coast. Soc Sci Med 2004;59:2547-60.

31 Rikkert MGMO, van den Bercken JHL, ten Have HAM, et al. Experienced consent in geriatrics research: a new method to optimize the capacity to consent in frail elderly subjects. J Med Ethics 1997;23:271-6.

32 Bayer A, Fish M. The doctor's duty to the elderly patient in clinical trials. Drugs Aging 2003;20:1087-97.

33 Fletcher JC. Ethical aspects of research involving elderly subjects. J Clin Ethics 1990;1:285-6.

34 Bjorn E, Rossel P, Holm S. Can the written information to research subjects be improved? An empirical study. J Med Ethics 1999;25:263-7.

35 Fitzgerald DW, Marotte C, Verdier RI, et al. Comprehension during informed consent in a less-developed country. Lancet 2002;360:1302-4. 